\title{
Research on of Optimization Design of Aluminum White Body Structure Based on Dynamics
}

\author{
Dan Wu \\ Automotive Engineering Department of Guangxi Traffic Vocational and Technical College, Nanning, \\ 530023
}

Keywords: aluminum white body structure; optimization design; dynamics

\begin{abstract}
The establishment of a finite element model that conforms to the actual assembly structure is the key to the dynamic optimization design of the structure. To this end, a three-dimensional CAD model of the white body of the Iveco A40 new passenger car has been established and the conversion to the finite element model has been achieved. Through the modal test of the white body, and the finite element model was modified to match the results of the finite element analysis with experimental test results. A finite element model that can reflect the dynamic characteristics of the actual body structure was obtained, laying the foundation for the structural optimization design of subsequent bodywork.
\end{abstract}

\section{Introduction}

The body is the key assembly of the cart. It is the driver's workplace and also the place where passengers and goods are accommodated. The bodywork should provide a convenient working environment for the driver, provide comfortable seating conditions for the occupants, protect them from the vibrations, noise, exhaust gas, and adverse weather conditions of the car, and ensure that the goods are carried intact and loaded. Structurally, its structure determines the shape of the vehicle, and more importantly, its mechanical properties can directly determine the dynamic characteristics of the entire vehicle (especially for the load-bearing body).

The body design can combine CAD technology with CAE dynamics modeling, static and dynamic analysis, sensitivity analysis, and dynamic parameter optimization in a virtual environment. Simulation replaces part of the prototype test. Before the prototype production, a comprehensive understanding of various dynamic and static performances to speed up new body development, improve development quality, and reduce development costs [1]. The body structure is made by spot welding a number of plate and shell parts with complex surfaces, therefore, it is very difficult to establish a white-body finite element model that can meet the actual structural characteristics.

The modal test obtains the dynamic characteristic parameters of the measured object from the actual structure, which can be used to modify the finite element model so that it can reflect the actual structural dynamic characteristics to perform the structural optimization design with engineering value. This is the test of the finite element model. Correcting technology has important application value in engineering [2]. The author takes the white body of Iveco A40 new bus as the object. Based on the 3D CAD model built by CATIA, the ANSYS software is used to build the white car body finite element model. , And according to the white body modal test results to modify the model built to lay the foundation for subsequent CAE analysis of the body.

The development of computer technology and the continuous improvement of mathematical theory have provided powerful tools for the structural analysis of automobiles. The finite element method is a commonly used structural strength analysis method that discards a closed form of analytical solution and seeks an approximate numerical solution that satisfies the actual needs of the project. However, the degree of credibility of the results obtained by finite element analysis is directly affected by the degree of agreement between the analytical model and the mechanical properties of the actual engineering structure. Therefore, the finite element model is modified through modal tests to make it more in line with the actual structure. This is currently an ideal way to get a high-precision model. 


\section{Build a White Body Finite Element Model}

Body 3D CAD model simplification and reconstruction The actual body-in-white 3D CAD model not only has many feature planes such as small holes, but each surface has its own individual (ie not shared with adjacent surfaces) contour lines. After the CAD model in CATIA was imported into ANSYS, after the free division, the local broken surface mesh was dense, the nodes on each side of the boundary contour line were not continuous, the total number of units was too much, and the calculation result was inaccurate. Therefore, to simplify the reconstruction model, The principle of simplification is to delete the assembly process holes, allowance steps, and transition fillets that have little effect on the mechanical properties of the structure; merge the facets into large faces, and share an outline between adjacent faces to ensure that each face is divided. The grids share nodes at the boundary so that there are no nodes staggered on the boundary. The result of this calculation maximizes the retention of the main mechanical characteristics of the part.

Meshing and unit quality inspection The A40 passenger car body is a lightweight, semi-loaded body with a light weight design. It is a shell structure with light weight and heavy load. The use of a housing unit to describe its mechanical properties is reasonable. Structural dynamic characteristics of Shell63 shell elements, side length is limited to $40 \mathrm{~mm}$, mainly quadrilateral elements. Element quality in the finite element model is critical to the analysis results, so the quality of the unit to be checked. The cell mass is related to the shell thickness. After checking the cell constants (part thickness), check the cell mass to avoid unnecessary duplication. The error cell should be deleted and replace the original warped quadrilateral cell by manually creating a new triangle cell [3].

\section{White Body Modal Test}

The degree of conformity between the built finite element model and the actual dynamic characteristics of the structure needs to be tested by tests, and it is gradually amended to match the experimental results. This is still an effective method for obtaining high-precision models.

During the test, the specially designed coil spring was used to lift the body to a free state. The natural frequency of the spring suspension system was far lower than the first-order natural frequency of the body-in-white. A total of 96 measurement points were arranged at the appropriate position on the white body. Using fixed-point hammer pulse excitation, picking up responses in multiple directions $(\mathrm{x}, \mathrm{y}, \mathrm{z})$. Using the hammering method, there is no additional mass and stiffness constraint on the tested part, the signal is basically attenuated within the sampling length, and energy leakage is less.

During data acquisition, an exponential window is added to the response signal to reduce the energy leakage. Each transfer function is repeatedly sampled and averaged several times to remove the random noise in the signal. After each sampling, observe the waveform of the transfer function and the coherence function. Under the normal condition of the waveform, the data with the coherence function greater than 0.8 at the peak is confirmed and saved.

\section{Error Analysis of Test and Analysis Results}

The main sources of error between the finite element analysis results and the test results are:

(1) Stiffness error. The spot welding joints between parts are lower in rigidity than the fixed joints. In the body model, the parts are fixed and connected, which is higher than the actual body spot welding, bolts and other joints [4].

(2) Mass error. The model simplification omits structures and small parts that have a small impact on the overall structural mechanical properties, and ignores the bolts and nut masses of bolted joints and other ancillary qualities such as paint and damping materials. Such finite elements The quality of the model is smaller than the actual quality.

(3) Errors in calculations and tests. In the process of discrete structures, iterative calculation processes, and signal acquisition and processing processes, unavoidable errors are generated. For the above analysis, the stiffness and mass distributions on the body finite element model are 
appropriately adjusted so that the finite element the model can meet the dynamic characteristics of the actual structure as much as possible, so that the calculation results and test results are fully close.

\section{Revision of White Body Finite Element Model}

Modal analysis is used to solve the vibration characteristics of a structure, including the natural frequency and mode shape. The natural characteristics of the structure can be obtained through modal tests. Based on the experimental results, according to the matrix perturbation reanalysis formula based on the linear generalized problem, it can be seen that each component of the natural frequency and natural mode of the vibration system varies with the structural mass and stiffness. In all cases of changing the position and modifying the position, find the most sensitive position for modifying the perturbation of the structure, so as to optimize the dynamics of the natural vibration characteristic of the structure so that the calculation result of the finite element model agrees with the test result.

Optimal Matrix Update: This correction method directly corrects the assembly mass and/or stiffness matrix to correlate the measured modality with the analytical modality. Recent developments in this approach include the use of constraints to maintain the connectivity of the model or to minimize matrix corrections. This topic uses this method before performing sensitivity analysis.

Sensitivity-based modal correction: This method modifies the finite element model using the derivatives of the behavioral parameters (or the sensitivity) of the model parameters. Compared to the optimal matrix correction (direct method), this method has the following advantages: the formula of the initial model, including its connectivity is implicitly saved; secondly, the results of the model modification can be represented by design parameters or errors in modeling assumptions. .

From the test results and calculation results, as well as the sensitivity analysis of the dynamic modification of the structure's intrinsic characteristics, careful adjustment of the connection stiffness between the parts and components and the mass distribution of the car body results in the built-in white-body finite element model and the actual white body. In this paper, the degree of conformity of the first three-step overall white body's natural frequency and vibration mode of the finite element model calculation and test is used as a criterion, and the parameters are modified and set to make it meet the ideal state. Correct the front and rear model calculation results and test results. The error between the theoretical calculation of the first three natural frequencies of the modified model and the experimental average is less than 3\%, and the mode shapes are consistent. This shows that the modified vehicle white-body finite element model and the actual structure are in mechanical properties, quality and the stiffness distribution has a high degree of compliance, which ensures that the analysis and optimization results based on this model will be credible [5].

The white body finite element model of the new Iveco A40 passenger car was established. Based on the modal test of the white body and based on the test results, the dynamic modification principle of the natural vibration characteristics of the structure was the theoretical guidance, and the white body finite element model was corrected. The obtained finite element model was obtained. Consistent with the dynamic characteristics of the actual vehicle body, it provides a theoretical basis for the subsequent analysis and optimization work of the vehicle body. The FEA modeling, modal test and the model correction method based on the test results introduced in the paper are also applicable to other mechanical structures.

\section{Model Corrections and Results}

A car body model is appropriately simplified, and a 4-node or 3-node space plate unit Shell63 is used to perform finite element mesh division. The body is discretely divided into 198121 nodes and 202056 units. Rigidity is used to connect parts and components. The pre-processing of the finite element model, without exerting any constraints and forces, left it in a free state. After performing 
the necessary parameter settings, the finite element model was analyzed using the algorithm in the ANSYS software to obtain the first six orders.

It is the joints that cause the modeling error. The modeling and the identification of physical parameters are topics that many scholars are concerned about. The joint surface recognition methods through computational and experimental correlation analysis can be divided into two categories: direct method and iterative method of model modification. The former is a series of vibration differential equations, derived from the modal parameters or frequency response function for solving the joint surface parameters of the formula, the method of damping derivation is complex and the identification error is large; the model modification of the joint recognition method is based on multiple iterations The optimization method has the characteristics of simple calculation, high accuracy and easy application (such as the use of various weighting methods, selection of different target variables, etc.), and it is a very effective method for identifying surface parameters. Among them, the use of universal custom units for modeling and simulation of the combined plane and complex boundaries has attracted the attention of many scholars. This method decomposes the stiffness matrix and the mass matrix at the cell level and the substructure level, and uses this as a response feature to correct the matrix of the cell or substructure by experimental data.

Considering the modality of testing the body-in-white in the free-free state, the method of suspending the body-in-white with a rubber rope was selected to approximate the free state. The natural frequency of the entire suspension system was lower than $2 \mathrm{~Hz}$. Excitation is achieved through an exciter. The exciter is controlled by a power amplifier and a pseudo-random signal is chosen to excite it. Due to the limited energy of a single point of excitation, for larger test objects, the results of measurement signals that are far from the excitation point often have large errors, which affects the accuracy of modal parameter identification. The author uses a two-point excitation method.

The grid division of the real car is to take a grid size of $100 \mathrm{~mm} \times 100 \mathrm{~mm}$, and the white body is divided as shown in Figure 3. The principle of measuring points is that the position of the external force, the important response point, the cross-linking point of the component or structure should generally be selected as the measuring point, the connection of the measured points should be able to display the shape of the body in white, and a total of 200 measurements are arranged on the body. Point, better define the body contour shape. The mode shape results of the modal tests are reflected by establishing a test geometry model. The test model should be able to fully reflect the contour of the vehicle body. The test model should be able to express the position of the actual measurement point conveniently and accurately.

\section{Conclusion}

The matrix shape method is used to directly modify the assembly quality and/or stiffness matrix so that the measured modality is related to the analytical modality, and the finite element model is modified based on the modal test results. The body structure is made up of several hundred parts connected by spot welding. Therefore, the connection interface characteristics between parts are the key to the dynamic characteristics of the entire vehicle body. Based on the experimental modal results, the finite element model of the actual vehicle structure, assembly relationship and welding state was repeatedly adjusted, such as adjusting the connection stiffness of the joints between the parts, so that the built-in vehicle body finite element model and the actual white body were Characteristically consistent.

\section{References}

[1] Yu Fan et al. Current status and development trend of automotive air suspension [J]. Automotive Technology, 2001, 8:6 -10

[2] Xu Lingzhen. Analysis of dynamic characteristics of automotive structures using modal experiments [J]. Hebei Institute of Technology Newspaper, 2004, 23(3):96-102. 
[3] Fu Zhifang. Theory and Application of Modal Analysis [M]. Shanghai: Shanghai Jiaotong University Press, 2000.157

[4] Xie Jun. Finite element modeling and dynamic analysis of automobile body [M]. Nanjing: Southeast University Academic Press, 2009.12

[5] Zhang Chengbao. Dynamic Analysis of Car Body Structure [J]. Automobile Research and Development, 2009(1): 25-27. 